Comparing 10 Methods for Solution Verification, and Linking to Model Validation

R. W. Logan, C. K. Nitta

March 25, 2005 
This document was prepared as an account of work sponsored by an agency of the United States Government. Neither the United States Government nor the University of California nor any of their employees, makes any warranty, express or implied, or assumes any legal liability or responsibility for the accuracy, completeness, or usefulness of any information, apparatus, product, or process disclosed, or represents that its use would not infringe privately owned rights. Reference herein to any specific commercial product, process, or service by trade name, trademark, manufacturer, or otherwise, does not necessarily constitute or imply its endorsement, recommendation, or favoring by the United States Government or the University of California. The views and opinions of authors expressed herein do not necessarily state or reflect those of the United States Government or the University of California, and shall not be used for advertising or product endorsement purposes.

This work was performed under the auspices of the U.S. Department of Energy by University of California, Lawrence Livermore National Laboratory under Contract W-7405-Eng-48. 
UCRL-TR-xxxxxx

\title{
Comparing 10 methods for Solution Verification, and Linking to Model Validation
}

\author{
Roger W. Logan ${ }^{*}$ and Cynthia K. Nitta \\ University of California \\ Lawrence Livermore National Laboratory \\ Livermore, CA 94551
}

\begin{abstract}
:
Grid convergence is often assumed as a given during computational analyses involving discretization of an assumed continuum process. In practical use of finite difference and finite element analyses, perfect grid convergence is rarely achieved or assured, and this fact must be addressed to make statements about model validation or the use of models in risk analysis. We have previously provided a 4-step quantitative implementation for a quantitative V\&V process. One of the steps in the 4-step process is that of Solution Verification. Solution Verification is the process of assuring that a model approximating a physical reality with a discretized continuum (e.g. finite element) code converges in each discretized domain to a converged answer on the quantity of subsequent validation interest. The modeling reality is that often we are modeling a problem with a discretized code because it is neither continuous spatially (e.g. contact and impact) nor smooth in relevant physics (e.g. shocks, melting, etc). The typical result is a non-monotonic convergence plot that can lead to spurious conclusions about the order of convergence, and a lack of means to estimate residual solution verification error or uncertainty at confidence. We compare ten techniques for grid convergence assessment, each formulated to enable a quantification of solution verification uncertainty at confidence and order of convergence for monotonic and nonmonotonic mesh convergence studies. The more rigorous of these methods require a minimum of four grids in a grid convergence study to quantify the grid convergence uncertainty. The methods supply the quantitative terms for solution verification error and uncertainty estimates needed for inclusion into subsequent model validation, confidence, and reliability analyses. Naturally, most such methodologies are still evolving, and this work represents the views of the authors and not necessarily the views of Lawrence Livermore National Laboratory.
\end{abstract}

Nomenclature

ANSI American National Standards Institute

ASME American Society of Mechanical Engineers

$\Delta \$ B \quad$ Benefit, usually in $\$ \$ \$$

$\triangle \$ B \quad$ Benefit, usually in $\$ \$ \$$

B Bias error estimate

BCR Benefit / Cost Ratio $(\Delta \$ \mathrm{~B}-\Delta \$ \mathrm{C}) / \Delta \$ \mathrm{C}$

$\Delta \$ C \quad$ Cost, usually in $\$ \$ \$$

C Confidence, a numerical value

CFD Computational Fluid Dynamics

E Bias error as percent of $F_{\mathrm{fg}}$

$\mathrm{F}_{\mathrm{fg}} \quad$ Quantity of Interest, discretized (computational) model solution, for finest grid

$\mathrm{F}_{\mathrm{i}} \quad$ Quantity of Interest, discretized (computational) model solution, for $\mathrm{i}^{\text {th }}$ grid

$\mathrm{F}_{\mathrm{i}}{ }^{*} \quad$ Quantity of Interest, response surface estimate solution, for $\mathrm{i}^{\text {th }}$ grid

$\mathrm{F}_{\mathrm{o}} \quad$ Quantity of Interest, exact solution

\footnotetext{
${ }^{*}$ Member, AIAA
} 


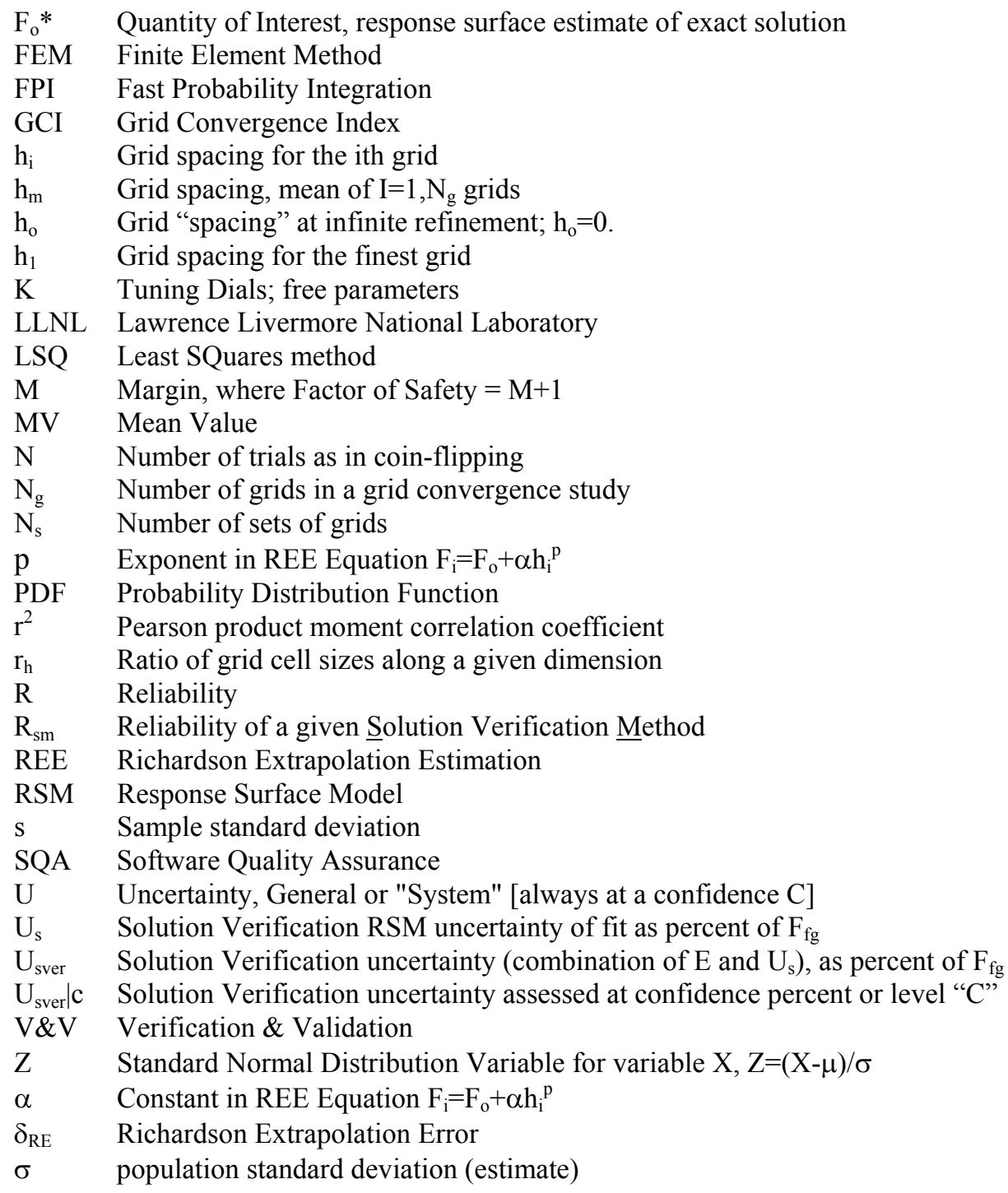

\section{Introduction}

The implementation of Verification and Validation $(\mathrm{V} \& \mathrm{~V})$ of a computational model of a physical system can be simply described as a 4-step “A-B-C-D' process":

First [A] is the planning and requirements phase. This includes description of the physical system (product) and its requirements, and a plan that takes into account what test data are available or can be obtained, what codes are available, and their status regarding software quality assurance (SQA) and Code Verification. A simple definition of Code Verification is that "verification means solving the equations right", i.e. if one intends to give an answer to the equation " $2+2=$ ", then one must run the resulting code to assure that the answer "4" results. If Code Verification or SQA are deemed insufficient for the importance of the problem to be modeled, Step [A] is the time to address these deficiencies.

Second of the four steps [B] is Solution Verification (the focus of this paper). Solution Verification is the process of assuring that a model approximating a physical reality with a discretized continuum (e.g. finite element) code converges in each discretized spatial, temporal, and iterative domain to a converged answer on the quantity of subsequent validation interest. We must assure ourselves that, with fine enough discretization and tight enough tolerances, our code is capable of obtaining the correct (i.e. $2+2=4$ ) answer; this is the realm of code verification. If 
we modeled finer and finer meshes and converged to $2+2=5$, with an order of convergence consistent with our numerical technique, we would recognize this as a code verification issue, but we might be happy regarding solution verification. So the process for solution verification would be to build a model, using a discretization that would provide, in our best judgment, a high quality answer for the quantity of interest in the regime of application of the model. The discretization should be made bearing in mind that we will want to refine or coarsen the grid to assess grid convergence and quantify the solution verification uncertainty. We can then run our discretized continuum model at finer and finer meshes, or, for a huge benefit in computational efficiency, run the model on coarser and coarser meshes, and obtain both a converged solution and a smooth order of convergence. The modeling reality is that often we are modeling a problem with a discretized code because it is neither continuous spatially (e.g. contact and impact) nor smooth in relevant physics (e.g. shocks, melting, etc). The typical result is a non-monotonic convergence plot that can lead to spurious conclusions about the order of convergence, and a lack of means to estimate residual error or uncertainty. We offer one emerging technique that enables a quantification of solution verification uncertainty at confidence $(\mathrm{U} \mid \mathrm{C})$ and order of convergence for monotonic and non-monotonic mesh convergence studies. In the ideal case, monotonic solution verification leads only to an error estimate term; a bias error. This bias error may be eliminated during the parameter estimation process inherent to some V\&V methods, but it should still be carried through as an additional uncertainty term in validation and reliability assessment. Nonmonotonic grid convergence adds an uncertainty of fit term, in addition to our treatment of the bias error estimate as an uncertainty. We will assess the solution verification uncertainty term, $U_{\text {sver, }}$, at a given level of statistical confidence, i.e. $\mathrm{C}=68 \%$ or $\mathrm{C}=95 \%$, etc. Unless otherwise stated, we will define $\mathrm{U} \mid \mathrm{C}$ where $\mathrm{C}=1$-sigma or $68 \%$ confidence (2-tailed) or $84 \%$ confidence (1-tailed) as assessed in this work with the assumption of a normal distribution. This will enable the $\mathrm{U}_{\text {sver }}$ term of Step [B] of our "ABCD" V\&V to be incorporated into the subsequent model validation, Step [C].

The third step [C] of our 4-step V\&V process is Model Validation over the domain of the available validation data referent. This involves the generation of both a mean and a confidence interval for model predictions, in light of comparison to the available referent data, and with inclusion of other uncertainty terms not directly accounted for in a simple model-vs.-data comparison. The error and uncertainty estimate from Solution Verification is one of these uncertainty terms; hence it must be quantified in a manner consistent with the other terms in this third step; that is, a bias or systematic error and an uncertainty at a specified confidence level.

The fourth step [D] of our 4-step V\&V process is the extrapolation of the third step away from the data domain into the domain of application; that is, the quantification of a predictive capability assessment ${ }^{2}$. The resulting confidence bounded uncertainty terms, from V\&V, may be used in combination with reliability methods to establish risk-based inputs into an adequacy or acceptance assessment ${ }^{3}$. Step [D] extends the model validation process of [C] out to the application domain of interest, which may be outside the domain of available data in one or more planes of multi-dimensional space. Even if the domain of application is "inside" the validation space from the referent data of Step "C", Step "D" must still justify the use of the physics, confidence intervals, and uncertainties, while interpolating within the space of the referent data in the validation domain. Step "D" should provide the numerical information about the model and its predictive capability such that given a requirement, an adequacy assessment can be made to determine if more computing capacity, code development, validation analyses or data are needed.

It has been said that $\mathrm{V} \& \mathrm{~V}$ must address tradeoffs for a "balance of sufficiency and efficiency" acknowledge (and ideally, quantify) the point when "better has become the enemy of good enough"”. These tradeoffs involve timing and funding for many issues including compute platforms, code development, analyses, and certification issues and schedules. In this work, we note that Solution Verification forms an important part of quantification of the tradeoff between speed of the computational analysis, and improvement in confidence. The improvement in confidence obtained due to a smaller assessed Solution Verification error and uncertainty estimate must be balanced against the cost of obtaining the additional compute capability to run the computational model at finer meshing. We have discussed such benefit / cost ratio tradeoffs in previous works in terms of the benefit / cost of doing additional code development or obtaining more experimental data ${ }^{5}$. In this paper, we will not extend our analysis to the benefit / cost of purchasing a larger compute platform based on our quantification of Solution Verification; but we will demonstrate and compare procedures to develop the terms to do just that. Naturally, most such methodologies are still evolving, and this work represents the views of the authors and not necessarily the views of Lawrence Livermore National Laboratory. 


\section{Grid Convergence in Solution Verification: Principles and Goals}

Step "B" in the 4-step "ABCD" validation process is that of Solution Verification. Solution Verification is the process of assuring that a model approximating a physical reality with a discretized continuum (e.g. finite element) code converges in each discretized domain to a converged answer on the quantity of subsequent validation interest. This is accomplished in the spatial domain by subdividing the elements or cells on the entire grid or portions of the grid. Grid convergence is only part of what is necessary to fully address Solution Verification, but we will limit the scope of our discussion to that part of the topic.

The first essential step in a grid convergence study is to plot the quantity of interest, $\mathrm{F}_{\mathrm{i}}$, versus the mesh spacing along a given direction, $h_{i}$, for a series of $\mathrm{I}=1, \mathrm{~N}_{\mathrm{g}}$ grids. (We will assume in this work that a best practice is to refine the grid simultaneously in each direction, although selective directional refinement can be useful in some instances.) To generate inputs to the subsequent validation, reliability, and risk processes, we will need outputs from solution verification in the form of (error+uncertainty) at a given confidence level. Our demand for a confidence level means that, since we have $\mathrm{K}_{\mathrm{g}}=3$ free parameters in our grid convergence model, we will find that in general, we need a minimum of $\mathrm{N}_{\mathrm{g}}=4$ grids, with $\mathrm{N}_{\mathrm{g}}>4$ highly desirable. Table 1 shows an example of a contrived grid convergence study, with $\mathrm{N}_{\mathrm{g}}=7$ grids, and 6 different series of grid convergence solution results, to represent the results that might be obtained from comparing 6 different codes or algorithms for solution. The exact solution is $\mathrm{F}_{\mathrm{o}}=600$. The first of the 6 grid sets is contrived to converge at $h_{o}=0$ to exactly $F_{o}=0$, with convergence order $p=2.0$. The remaining 5 grid sets are perturbed slightly. In this sense, all of the latter 5 grid sets will yield a non-monotonic grid convergence exponent $\mathrm{p}$, since the output quantity $\mathrm{F}_{\mathrm{i}}$ is non-smooth. In the extreme, even the output quantity $\mathrm{F}_{\mathrm{i}}$ may be nonmonotonic, and at this time some traditional grid convergence approaches will fail entirely. Typically such results are plotted first on a linear scale as in Figure 1, then on a $\log -\log$ scale as $\log$ assessed error $\left(\mathrm{F}_{\mathrm{i}}-\mathrm{F}_{\mathrm{o}}\right)$ vs. $\log$ grid spacing.

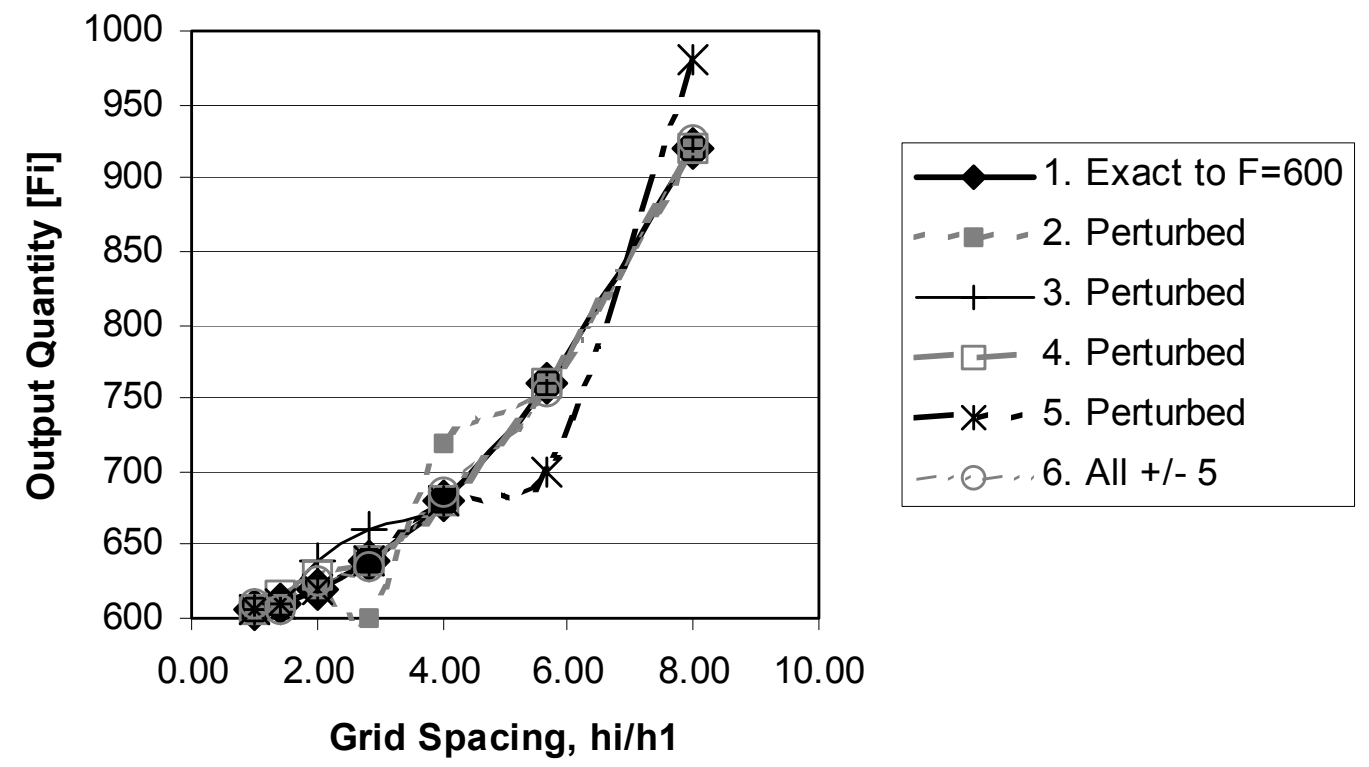

Figure 1. Plots that begin a grid convergence study. Quantity of interest $F_{\mathrm{i}}$ vs grid spacing $\mathbf{h}_{\mathrm{i}}$, on linear scale. Exact second order " $p=\mathbf{2 . 0 0 " ~ r e s u l t s ~ w i t h ~ p e r t u r b a t i o n s ~ t o ~ t e s t ~ a s s e s s m e n t ~ o f ~ s o l u t i o n ~ v e r i f i c a t i o n ~}$ uncertainty. 
TABLE I. DATA FOR "EXACT" GRID CONVERGENCE SET: $\mathrm{F}_{\mathrm{o}}=600$ with $\mathrm{p}=\mathbf{2 . 0}$

\begin{tabular}{|c|c|c|c|c|c|c|}
\hline hilh1 & 1. Exact to $F=600$ & 2. Perturbed & 3. Perturbed & 4. Perturbed & 5. Perturbed & 6. All +-5 \\
\hline 8.000 & 920.00 & 920.00 & 920.00 & 920.00 & 980.00 & 925.00 \\
\hline 5.657 & 760.00 & 760.00 & 760.00 & 760.00 & 700.00 & 755.00 \\
\hline 4.000 & 680.00 & 720.00 & 680.00 & 680.00 & 680.00 & 685.00 \\
\hline 2.828 & 640.00 & 600.00 & 660.00 & 640.00 & 640.00 & 635.00 \\
\hline 2.000 & 620.00 & 620.00 & 640.00 & 630.00 & 620.00 & 625.00 \\
\hline 1.414 & 610.00 & 610.00 & 610.00 & 615.00 & 610.00 & 605.00 \\
\hline 1.000 & 605.00 & 605.00 & 605.00 & 605.00 & 605.00 & 610.00 \\
\hline
\end{tabular}

Figure 1 has features typical of a grid convergence study. It looks like our quantity of interest $\mathrm{F}_{\mathrm{i}}$ converges to $\mathrm{F}_{\mathrm{o}} \sim 600$, but how can we be sure? What bias error and uncertainty can we assess given that we may only have $\mathrm{F}_{\mathrm{fg}}$, our finest grid solution? We want to know how far we are from the exact solution $F_{0}=600$, which is known in this case but unknown in general. There are many assumptions often made during grid convergence studies ${ }^{6}$, leading to the frequent use of the Richardson Extrapolation Estimation (REE) technique ${ }^{7}$. The REE method assumes that the grid convergence results are in the asymptotic convergence regime, neglects higher order terms in its approximation assumptions, and assumes that convergence of the discretized model solutions $F_{i}$ is of the form ${ }^{8}$ :

$\mathrm{F}_{\mathrm{i}}=\mathrm{F}_{\mathrm{o}}+\alpha \mathrm{h}_{\mathrm{i}}^{\mathrm{p}}$

We will build a Least-Squares (LSQ) or Response Surface Method ( $\left.\mathrm{RSM}^{9}\right)$ model to provide monotonic solutions approximating the discretized solutions. We construct the RSM solutions $\mathrm{F}_{\mathrm{i}}{ }^{*}$ of the same form:

$\mathrm{F}_{\mathrm{i}}^{*}=\mathrm{F}_{\mathrm{o}}{ }^{*}+\alpha \mathrm{h}_{\mathrm{i}}^{\mathrm{p}}$

In Eqn. [1], $\mathrm{F}_{\mathrm{i}}{ }^{*}=$ the solution from the $\mathrm{i}^{\text {th }}$ grid (either the code result $\mathrm{F}_{\mathrm{i}}$, or a Least-Squares (LSQ) or Response Surface Method (RSM) approximation $\mathrm{Fi}^{*}$ ). If the grid convergence study is perfectly monotonic with constant convergence exponent ' $\mathrm{p}$ ', we will have $\mathrm{F}_{\mathrm{i}}=\mathrm{F}_{\mathrm{i}}{ }^{*}$ for all grids. We will see that this is rarely the case except in contrived examples. We desire to have or obtain $\mathrm{F}_{\mathrm{o}}=$ the exact known solution, or $\mathrm{F}_{\mathrm{o}}{ }^{*}=$ the estimate of the exact solution, where $\mathrm{h}_{\mathrm{i}}=$ the ith grid spacing, $\mathrm{p}=$ the convergence order (either theoretical or fitted), and $\alpha=\mathrm{a}$ fitting constant.

We first obtain an estimate of the exponent $\mathrm{p}$ expressing the order of convergence to the fully converged solution $\mathrm{F}_{\mathrm{o}}$. For any grid triplet or series of three grids, with a constant $\mathrm{p}$ and grid refinement ratio " $\mathrm{r}_{\mathrm{h}}$ ", we can calculate the order of convergence (exponent $\mathrm{p}$ ), neglecting higher order terms, as:

$\mathrm{p}=\log \left[\left(\mathrm{F}_{1}-\mathrm{F}_{3}\right) /\left(\mathrm{F}_{1}-\mathrm{F}_{2}\right)\right] / \log \left[\mathrm{r}_{\mathrm{h}}\right]$

Eqn. [2] and subsequent equations follow from Eqn. [1] and hence carry the same caveats and assumptions. There are more general methods as discussed by Roache ${ }^{6}$ that do not require a constant $r_{h}$. If the model results are consistent with an exact convergence order exponent, we can use any combination of grid results for the quantity of interest $F_{i}$ in Eqn. [2] and obtain the same exponent p. We could then calculate an estimate of the exactly converged solution as in Roache ${ }^{6}$ as:

$\mathrm{F}_{\mathrm{o}}{ }^{*}=\mathrm{F}_{\text {exact }}=\mathrm{F}_{1}+\left(\mathrm{F}_{1}-\mathrm{F}_{2}\right) /\left(\mathrm{r}_{\mathrm{h}}{ }^{\mathrm{p}}-1\right)$

If this is true, we will obtain correlation coefficient $r^{2}=1$ to the regression of the line fitting $\log \left(\mathrm{F}_{\mathrm{i}}{ }^{*}-\mathrm{F}_{\mathrm{o}}{ }^{*}\right)$ versus $\log \left(\mathrm{h}_{\mathrm{i}}\right)$. We will also obtain a sample standard deviation $\mathrm{s}=0$ (and bias $\mathrm{B}=0$ ) on comparison of the set of computational model results and regression fit results. However, even with a mesh convergence study giving model results that are quite smooth, it is almost never the case that all the $F_{i}$ in the study will yield exactly the same exponent $\mathrm{p}$. For example, to obtain exactly $\mathrm{p}=2$ at all points in a grid convergence study, we would need to be modeling a problem whose exact solution was a quadratic, with a numerical formulation capable of capturing a quadratic solution exactly. Therefore to obtain an estimate of $p$ over the entire domain of grid sizes, we suggest a regression fit "response surface model" (RSM) to the linearization of (Eqn. [1]) to obtain a regression slope p. 
We can obtain a regression value of $\mathrm{p}$, with a correlation coefficient (hopefully $\mathrm{r}^{2} \sim 1$ ), and standard deviation comparing the regression fit to the computational model grid refinement ratios. We can estimate the error in our finest grid solution $\left(F_{1}\right)$ using Eqn. 2. This value, in addition to a systematic error or bias estimate, may also be taken as an uncertainty. The proper choice here is not universally established in the community, since in application of the model, we cannot always assure ourselves of the direction of the bias error; only that we have one at any grid more coarse than the exact $\mathrm{F}_{\mathrm{o}}$. Therefore we might take the bias error and also sum in quadrature ${ }^{10}$ the uncertainty taken from the Richardson Extrapolation Estimation (REE), with the uncertainty taken from the (small sample corrected) standard deviation of the computational model result minus the regression fit (response surface model) at constant p. Or, we might simply take a linear sum of the REE estimate, (E) and the response surface model (RSM) uncertainty estimate, $\left(\mathrm{U}_{\mathrm{s}}\right)$. When the bias error term B, or E as we denote it for the Richardson Extrapolation term, is taken as an additional uncertainty, we choose in this work to scale $\mathrm{E}$ to $\mathrm{E}_{\mathrm{C}}$, scaled from $\mathrm{E}$ by the statistically assessed percent confidence used for the rest of the analysis. The ASME / ANSI standard quadrature process ${ }^{10}$ essentially defines use of the entire $100 \%$ of the bias as an uncertainty (B), in quadrature with a normal 2 -sigma or $95 \%$ confidence uncertainty $\mathrm{S}$, or $\mathrm{U}_{\mathrm{s}}$ in our notation. Our procedure uses a normal 1-tail 2-sigma fraction $97.7 \%$ of the bias error $\mathrm{B}$ (or $\mathrm{E}_{\mathrm{C}}=0.977 \mathrm{E}$ here) in quadrature with $\mathrm{U}_{\mathrm{s}}$ at 2-sigma, which is essentially the same as the ASME standard $^{10}$. For a 1-sigma analysis, we combine the 1-tailed $84 \%\left(\mathrm{E}_{\mathrm{c}}=0.841 \mathrm{E}\right)$ with $\mathrm{U}_{\mathrm{s}}$ at 1 -sigma. We are not aware of a well accepted standard for combining model assessed bias (B or E) with model fit uncertainty $\left(\mathrm{U}_{\mathrm{s}}\right.$ here) and would welcome such a standard. Meanwhile, we are exploring more rigorous treatments of the bias terms when treated as added uncertainties. The procedure we suggest and use here does not greatly influence our results or conclusions, but this may not always be the case. We will use the linear combination $\left(E_{c}+U_{s}\right)$ in part of this work as noted, but unless otherwise stated, this work will use the root-sum-squares combination to obtain an estimate of the total Uncertainty $(\mathrm{U})$ from our grid convergence study:

$\mathrm{U}=\left(\mathrm{E}_{\mathrm{c}}^{2}+\mathrm{U}_{\mathrm{s}}^{2}\right)^{(1 / 2)}$

This quantity $(\mathrm{U})$ is expressed as a percent of the estimated exact solution $\mathrm{F}_{\mathrm{o}}$. For a smooth, monotonic example, $\mathrm{U} \sim 0$ since the regression fit response surface is essentially an exact duplicate $\left(\mathrm{r}^{2}=1\right)$ of the computational model, so the entire error " $\mathrm{E}_{\mathrm{c}}+\mathrm{U}_{\mathrm{s}}$ " is just " $\mathrm{E}_{\mathrm{c}}$ ", the REE estimate taken as a confidence scaled bias uncertainty for the fine grid, after Roache ${ }^{6}$ :

$\mathrm{E}_{1}[$ fine grid $]=\left(\mathrm{F}_{2}-\mathrm{F}_{1}\right) /\left(\mathrm{r}_{\mathrm{h}}{ }^{\mathrm{p}}-1\right)$

Eça and Hoekstra ${ }^{8}$ define the REE as

$\delta_{\mathrm{RE}}=\mathrm{E}_{1}=\mathrm{F}_{\mathrm{i}}{ }^{*}-\mathrm{F}_{\mathrm{o}}{ }^{*}=\alpha \mathrm{h}_{\mathrm{i}}^{\mathrm{p}}$

For the finest of the $\mathrm{I}=1, \mathrm{~N}_{\mathrm{g}}$ grids $(\mathrm{I}=\mathrm{fg})$ we have:

$\delta_{\mathrm{RE}}=\mathrm{E}_{1}=\mathrm{F}_{\mathrm{fg}}{ }^{*}-\mathrm{F}_{\mathrm{o}} * \alpha \mathrm{h}_{\mathrm{fg}}^{\mathrm{p}}$

We report percent error estimate $\delta_{\mathrm{RE}}$ or $\mathrm{E}$ as a percent of the exact solution or its estimate $\mathrm{F}_{\mathrm{o}}$ *:

$\mathrm{E}=\mathrm{E}_{1} *(100 \%) / \mathrm{F}_{\mathrm{o}} *$

As noted above, it is rare that a grid convergence study of $\mathrm{i}=1, \mathrm{~N}_{\mathrm{g}}$ grids will exhibit an exact fit to these equations, with $\mathrm{p}=$ constant and no oscillations. To address this, the three free parameters $\mathrm{K}_{\mathrm{g}}=3,\left(\mathrm{~F}_{\mathrm{o}}{ }^{*}, \alpha\right.$, and $\left.\mathrm{p}\right)$ are best-fit to the grid convergence study of $\mathrm{I}=1, \mathrm{~N}_{\mathrm{g}}$ grids. To account for the fitting process, Eça and Hoekstra ${ }^{8}$ also include a Least -Squares (LSQ) uncertainty term, which Nitta and Logan ${ }^{3}$ call the Response Surface Method (RSM) uncertainty term:

$\mathrm{U}_{\mathrm{s}}=\sqrt{\sum_{\mathrm{I}=1}^{\mathrm{Ng}}\left(\mathrm{F}_{\mathrm{i}}-\left(\mathrm{F}_{\mathrm{o}} *+\alpha \mathrm{h}_{\mathrm{i}}^{\mathrm{p}}\right)\right)^{2} /\left(\mathrm{N}_{\mathrm{g}}-\mathrm{K}_{\mathrm{g}}\right)}$ 
Our estimate of solution verification (SVER) grid convergence uncertainty $\mathrm{U}=\mathrm{U}_{\text {sver }}$ (at a given confidence level) given by Eqn. [4] is the term that we can use as a Mean Value (MV) or other Fast Probability Integration (FPI) method term in subsequent model validation, reliability, risk, and benefit / cost quantification. Therefore, the body of this work will describe various ways, from simple to elaborate, to obtain $\mathrm{U}_{\text {sver }}$ estimates, and how these methods behave on several challenging (oscillatory or non-monotonic) grid convergence examples.

\section{Grid Convergence in Solution Verification: Methods}

We now describe, using the notation above, a range of methods from simple to elaborate, to obtain a quantified estimate of $U=U_{\text {sver }}$, the solution verification grid convergence uncertainty estimate of Eqn. [4]. There are 10 such methods, each building on the others, that we will describe:

1. For a series of grids $\mathrm{I}=1, \mathrm{~N}_{\mathrm{g}}$ that is reasonably smooth and monotonic, use the Grid Convergence Index (GCI) method as described by Roache ${ }^{6}$ and others. We realize that "reasonably" is a very subjective term. For Computational Fluid Dynamics (CFD) problems, there are good and suggested correlations whereby the basic REE estimate is multiplied by a GCI factor of safety, $\mathrm{Fs}=1.25$ for $\mathrm{N}_{\mathrm{g}}=3$ or more, and $\mathrm{F}_{\mathrm{s}}=3.00$ for $\mathrm{N}_{\mathrm{g}}=2$. With these $F_{s}$ in the GCI, one can obtain an estimate of $U=U_{\text {sver }}$ at what is contended to be $95 \%$ confidence ${ }^{6}$. The use of these $F_{s}$ with the contention of $95 \%$ confidence is based on correlation with an extensive database of agreement for CFD. It is not clear that the same $95 \%$ confidence level correlates with the use of these same $\mathrm{F}_{\mathrm{s}}$ for computational mechanics or other finite element, finite difference, or finite volume grid studies.

2. Use the GCI process described as method $\# 1$, with $\mathrm{F}_{\mathrm{s}}=1.25$ and $\mathrm{N}_{\mathrm{g}}=4$ or more. Take the resulting $\mathrm{U}_{\mathrm{sver}}=\mathrm{U}$ as a $68 \%$ confidence estimate. We suggest this procedure because, based on our small but growing database of nonsmooth grid convergence studies, a claim of $68 \%$ confidence fits much better than a claim of $95 \%$ confidence. We will show examples of this below.

Method \#1 and method \#2 will work well for smooth, monotonic grids where the physics and mathematics of the problem actually yield a constant, or even approximately constant ${ }^{11}$, order $\mathrm{p}$ of convergence for the problem at hand. However, method \#1 and method \#2 will fail for non-monotonic grid convergence results. This is because these methods require terms of $\log \left(\right.$ error), such as $\log \left(\mathrm{F}_{\mathrm{i}}-\mathrm{F}_{\mathrm{i}-1}\right)$ or $\log \left(\mathrm{F}_{\mathrm{i}}-\mathrm{F}_{\mathrm{o}}{ }^{*}\right)$ to obtain estimates of the slope $p$ in Eqn. [1]. Because of this, we suggest the next three methods. For each of these methods, \#3, \#4, and \#5, the first step is to guess a value for $\mathrm{p}$ in Eqn. [1] and then plot, on a linear scale, the observed grid solutions Fi versus $h_{i}^{p}$. The intercept is then $\mathrm{F}_{\mathrm{o}}{ }^{*}$, and the slope is $\alpha$ in Eqn. [1].

3. For this method, we suggest iterating to find $p$ that maximizes the correlation coefficient $r^{2}$ of the linear fit of $F_{i}$ versus $h_{i}^{p}$. Then, use a safety factor $F_{s}=3$ and take the resulting GCI (Eqn. [7] multiplied by $F_{s}$ ) as the 1-sigma estimate of $U_{\text {sver }}=U$. The value of $F_{s}=3$ with 1-sigma may seem extreme, but with our limited data base of nonsmooth grid studies with exact solutions, it is the best fit we have so far.

4. Method \#4 is one step simpler than Method \#3. Plot $F_{i}$ versus $h_{i}{ }^{p}$, choosing first $p=1$ and then $p=2$. Take the maximum of the two uncorrected $\delta_{\mathrm{RE}}=\mathrm{E}_{1}$ (E as a percent) as the 1-sigma estimate of $\mathrm{U}_{\mathrm{sver}}=\mathrm{U}$.

5. This method is a variant on Method \#4. Obtain $\delta_{\mathrm{RE}}=\mathrm{E}_{1}$ as in method $\# 4$ for both $\mathrm{p}=1$ and $\mathrm{p}=2$, and take the average of these same two uncorrected $\delta_{\mathrm{RE}}=\mathrm{E}_{1}$ as the 1-sigma estimate of $\mathrm{U}_{\mathrm{sver}}=\mathrm{U}$.

Methods \#1-\#5 are either well described in the literature (\#1 and \#2) or they are robust and simple to implement (\#3, \#4, and \#5). They have the advantage that Method \#1 and \#2 are obtained together with no 
additional work, and have been used with as few as $\mathrm{N}_{\mathrm{g}}=2$ grids, although we know of no one in the community advocating $\mathrm{N}_{\mathrm{g}}=2$. In defense of $\mathrm{N}_{\mathrm{g}}=2$ or even $\mathrm{N}_{\mathrm{g}}=3$ for simulations of low risk scenarios, we recognize that generating the meshes and grids appropriate for solution verification is one of the most challenging and labor-intensive tasks in the finite element community today. (We also recognize that all too often, we see the use of $\mathrm{N}_{\mathrm{g}}=2$ or $\mathrm{N}_{\mathrm{g}}=3$, or even $\mathrm{N}_{\mathrm{g}}=1$ for simulations that could hardly be regarded as applied to "low risk" scenarios. Method \#4 and Method \#5 are also obtained together with no additional work. A few more iterations (on the guess for $\mathrm{p}$ ) will give Method \#3. These first 5 methods are so simple that we suggest looking at them as a first simple check on the number being used for $\mathrm{U}_{\text {sver }}=\mathrm{U}$. Method \#6 through Method \#10 all involve generation of an uncertainty term $U_{s}$ explicitly. To do this, there is a minimum requirement that $\mathrm{N}_{\mathrm{g}}>3$, since we have $\mathrm{K}=3$ free parameters $\left(\mathrm{F}_{\mathrm{o}}{ }^{*}, \alpha, \mathrm{p}\right)$ to fit. Four grids $\mathrm{N}_{\mathrm{g}}=4$ is a minimum needed, and even $\mathrm{N}_{\mathrm{g}}=4$ will lead to use of fairly large small sample corrections with the many assumptions inherent to small sample statistics. Our examples contain studies with $\mathrm{N}_{\mathrm{g}}=6$ and $\mathrm{N}_{\mathrm{g}}=7$. However, an application specific grid convergence study with $\mathrm{N}_{\mathrm{g}}=6$ or more is rare, especially for complex problems with complex geometry.

6. Method \#6 is the method exactly as described in Eça and Hoekstra ${ }^{8}$ and hence denoted the EH04 method here. Of note, the estimate of $\mathrm{E}$ is obtained by multiplying $\delta_{\mathrm{RE}}$ by a GCI $\mathrm{F}_{\mathrm{s}}=1.25$, which is often taken as $95 \%$ confidence. This is added to $U_{s}$ at 1-sigma to obtain $U_{\text {sver }}=U$. We will call this a 1-sigma estimate of $U_{\text {sver }}=U$ in the examples below. However, as discussed above, there is no standard or consensus on combining bias error style uncertainty terms $\left(\delta_{\mathrm{RE}}\right.$ or $\mathrm{E}$ or B in general) with model fit uncertainty or variability terms $\left(\mathrm{U}_{\mathrm{s}}\right.$ in this case). Of note is in the Eça and Hoekstra EH04 method, the fit is obtained by choosing the K=3 free parameters to minimize the $\mathrm{U}_{\mathrm{s}}$ of Eqn. [9].

7. Method \#7 was developed and implemented independently by Nitta and Logan ${ }^{9}$ (denoted NL04u) and in about the same time frame as the EH04 method. Both methods consist of a process that has logical elements and both are very similar except for certain details. In the NL04 method, we do not currently use the GCI style $\mathrm{F}_{\mathrm{s}}=1.25$ but rather $\mathrm{F}_{\mathrm{s}}=1.00$. However, we do use a small sample correction (Student's $t$ or other depending on conservatism) based on the number of grids $=\mathrm{N}_{\mathrm{g}}$, and the number of free parameters $\mathrm{K}=3$. Like the EH04 method, we perform a least squares fit to minimize the term $U_{s}$ in Eqn. [9].

8. Method \#8 is an extension of Method \#7, also from Nitta and Logan ${ }^{9}$, and is denoted NL04eu. The procedure is identical to Method \#7 (NL04u), except that we perform the least squares procedure to generate a response surface model of the code results on the $\mathrm{I}=1, \mathrm{~N}_{\mathrm{g}}$ grids, this time to minimize the total term $\mathrm{U}_{\text {sver }}=\mathrm{U}$ in Eqn. [4]. That is, instead of minimizing $U_{s}$ of Eqn. [9], we minimize either the linear or quadrature combination of $E$ with $\mathrm{U}_{\mathrm{s}}$. This does not seem as faithful to the principles of REE or even LSQ REE with RSM as does the NL04u or EH04 method. However, as we noted on some non-monotonic examples, minimizing $U_{s}$ in Eqn. [9] can give spurious results for the free parameters $\mathrm{K}=\left(\mathrm{F}_{\mathrm{o}}{ }^{*}, \mathrm{a}, \mathrm{p}\right)$. The NL04eu method is far more stable in these situations. The NL04eu procedure of Method \#8 will give slight errors in the case of an exactly correct grid convergence study, compared to the NL04u or EH04 methods which will converge to a fit with $\mathrm{U}_{\mathrm{s}}=0$ and $\mathrm{F}_{\mathrm{o}}{ }^{*}=\mathrm{F}_{\mathrm{o}}$, the exact analytical solution. For example, consider the grid convergence in the left column of Table 1 . This contrived set of solutions gives the exact $(\mathrm{p}=2.000)$ expected answer as the grid is refined, with perfect convergence predicted to $\mathrm{F}_{\mathrm{o}}{ }^{*}=\mathrm{F}_{\mathrm{o}}=600$. Both the $\mathrm{NL} 04 \mathrm{u}$ and $\mathrm{EH} 04$ procedures will converge to $\mathrm{F}_{\mathrm{o}}{ }^{*}=\mathrm{F}_{0}=600$, and provide correct estimates of discretization error $\mathrm{E}$ for the finest grid used (bottom row of Table 1, e.g. $\mathrm{F}_{\mathrm{fg}}=605$ vs. $\mathrm{F}_{\mathrm{o}}{ }^{*}=\mathrm{F}_{\mathrm{o}}=600$ ). To show that even in this situation of perfect grid results for perfect $\mathrm{P}=2.000$ convergence, the approximation of the NL04eu is a good one, we used both NL04u and NL04eu on this exact grid study. We used progressively coarser values for hi at $\mathrm{I}=\mathrm{Ng}$, the finest grid, and compared the error in the estimate of $\mathrm{F}_{0}=600$ or $\mathrm{F}_{0}=100 \%$ (known exactly), in light of the known ratio of the fine grid solution $\mathrm{F}_{\mathrm{fg}} / \mathrm{F}_{\mathrm{o}}$. We also compared the overall $U_{\text {sver }}=U$, the quadrature combination of $E$ and $U_{s}$ (Eqn. [4]). Figure 2 shows that at fine grids, the NL04u and NL04eu methods are indistinguishable. At more coarse values of $h_{i}, I=N_{g}$, we see that while NL04u continues to converge to the exact $\mathrm{F}_{0} * / \mathrm{F}_{0}=100 \%$, NL04eu drifts off the exact solution, giving $\mathrm{F}_{\mathrm{o}} * / \mathrm{F}_{0}=104 \%$ for very coarse grids. However, at this same grid, we have $\mathrm{F}_{\mathrm{fg}} / \mathrm{F}_{\mathrm{o}}=153 \%$. We feel that the robustness of the NL04eu is worth tolerating this small difference. Similarly, Figure 3 shows a comparison of $U_{\text {sver }}=U$, the quadrature of $E$ and $U_{s}$, for both the NL04u and NL04eu methods. At the coarse grid, the $\mathrm{U}_{\text {sver }}$ estimate is $\mathrm{U}=109 \%$ for NL04u, and only $\mathrm{U}=105 \%$ for NL04eu. Both of these estimates of $\mathrm{U}$ (at 1-sigma confidence) are so large as to make the grid 
choice practically useless. And still, the NL04u and NL04eu methods are very close in their estimate of $F_{\mathrm{o}}{ }^{*} \sim 600$ and the assessed $U_{\text {sver }}$. Therefore, given that we observe that the discrepancy created by using the NL04eu method is small, we strongly recommend using both Method \#7 and Method \#8. Method \#7 (NL04u) is more nearly correct in the exact limit (but is still subject to the approximations of Richardson Extrapolation anyway). Method \#8 (NL04eu) is an essential partner because it will alert us to spurious results that may result from NL04u in non-smooth grid convergence studies. As shown in Fig. 2 and Fig. 3, there should not be much difference between the $\mathrm{U}_{\text {sver }}$ obtained from Method \#7 and Method \#8. If there is, we suggest using the larger of the two $\mathrm{U}_{\text {sver }}$ so obtained while the reason for the large discrepancy is investigated.

9. Method \#9 is identical to Method \#7 with one important addition. In Method \#9 (denoted LN05u), we note that, consistent with the original intent of the GCI, we are extrapolating our grid convergence study, with $\mathrm{h}(\mathrm{I}=$ $\mathrm{N}_{\mathrm{g}}$ ) the coarsest grid, and $\mathrm{h}(\mathrm{I}=1)$ the finest grid, to $\mathrm{h}_{0}=0$, the estimate $\mathrm{F}_{\mathrm{o}}{ }^{*}$ of the fully converged solution $\mathrm{F}_{\mathrm{o}}$. Beginning with the EH04 and NL04 methods, we are now mixing in the statistics of a least squares uncertainty term $U_{s}$. In the NL05u method, we note this, and as part of our extrapolation to $h_{0}=0$, we correct with a statistical multiplier for the prediction interval at $h_{0}=0$. This is the simplest of standard parabolic corrections ${ }^{12}$ that is a function of the interval from $\mathrm{h}_{\mathrm{fg}}(\mathrm{I}=1)$ to $\mathrm{h}_{\mathrm{cg}}\left(\mathrm{I}=\mathrm{N}_{\mathrm{g}}\right)$, with mean $\mathrm{h}_{\mathrm{m}}$. The prediction interval correction is a function of distance from $h o=0$ to the mean of the grids used, $h_{i}=h_{m}$, compared to the span of the coarsest hi=hcg and finest $h_{\mathrm{i}}=\mathrm{h}_{\mathrm{fg}}$ grids used. This extrapolation prediction interval estimate will be small if $h_{\mathrm{i}}=\mathrm{h}_{\mathrm{fg}}$ is close to $\mathrm{h}_{\mathrm{o}}=0$ already, and large if $h_{\mathrm{i}}=\mathrm{h}_{\mathrm{fg}}$ is far from $\mathrm{h}_{\mathrm{o}}=0$.

10. Method \#10 is identical to Method \#9, except that we minimize the linear or quadrature combination of $\mathrm{E}+\mathrm{U}_{\mathrm{s}}$, so we denote this method as (LN05eu). Method \#9 and \#10 (LN05u and LN05eu) form a pair in that both use the prediction interval correction. Method \#7 and \#8 (NL04u and NL04eu) form an identical pair, except that they do not use the prediction interval correction. Method \#9 and \#10 obtain the prediction interval (P.I.) by multiplying the term Us by:

P.I. $=\mathrm{U}_{\mathrm{s}} * \sqrt{1+\frac{1}{\mathrm{Ng}}+\frac{\left(0-\mathrm{h}_{\mathrm{m}}\right)^{2}}{\left.\sum_{\mathrm{i}}\left(\mathrm{h}_{\mathrm{i}}-\mathrm{h}_{\mathrm{m}}\right)\right)^{2}}}$

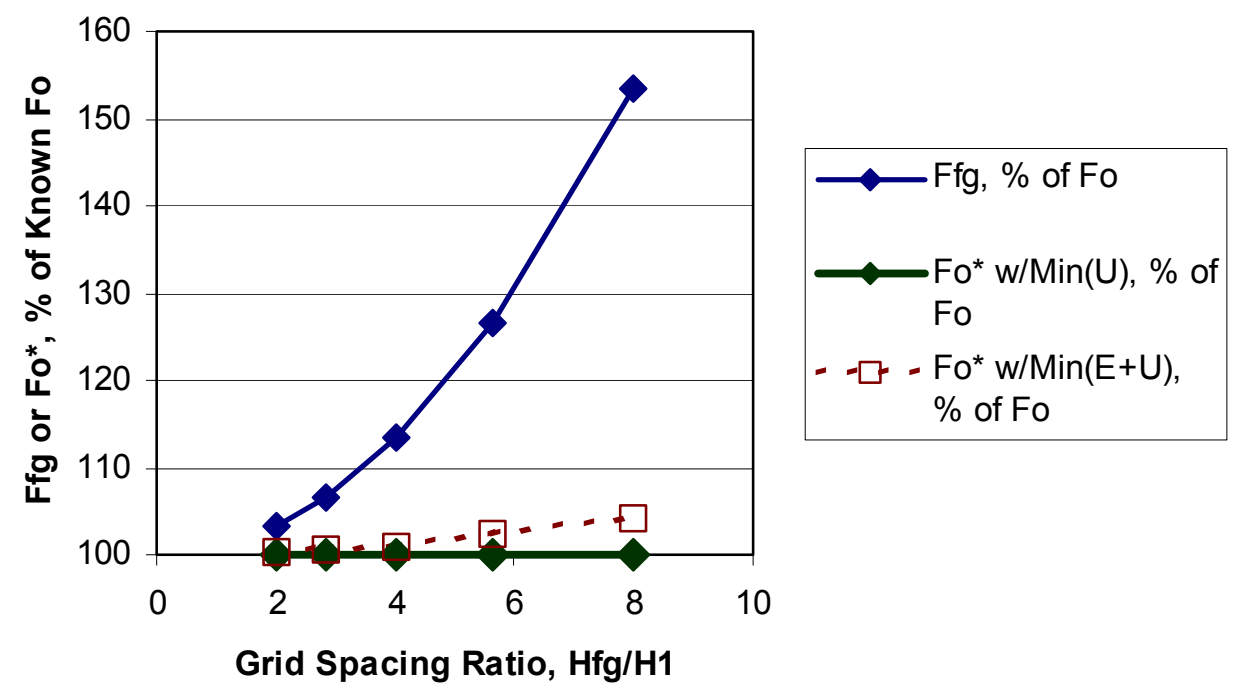

Figure 2. Comparison of the estimate of $F_{0} * / F_{0}$ for Method $\# 7$ and Method $\# 8$ (more robust but approximate). Both estimates are excellent compared to the error in $F_{\mathrm{fg}} \mathbf{v s} F_{0}$ for the very "coarse" choices of finest grid spacing $\mathbf{H}_{\mathrm{fg}}$. 


\section{Grid Convergence in Solution Verification: Results and Reliability}

To compare each of the 10 methods that we suggest for quantification of the grid convergence portion of solution verification uncertainty at confidence, we compared each of the 10 methods on grid convergence studies that had known solutions. The first of these was the set of grid convergence results given in Table 1 . This study has $\mathrm{N}_{\mathrm{g}}=7$ grids, with $\mathrm{N}_{\mathrm{s}}=6$ sets of results for comparison. The second series was taken from the $2^{\text {nd }}$ Verification Suite, a comparison by Harrison and Conway ${ }^{13}$ at LLNL. This annual Verification Suite was begun as part of our first edition V\&V Methodology ${ }^{14}$ several years ago, with results first reported in the $1^{\text {st }}$ Verification Suite by Sam et al ${ }^{15}$. This second series contains a grid convergence study with $\mathrm{N}_{\mathrm{g}}=7$ grids, for a beam bending problem. We compare to the exact solutions for bending stress and end deflection for shell elements. The beam bending model is a discretized model of a beam assumed to follow the governing equation:

$$
\operatorname{EI}\left(d^{2} y / d x^{2}\right)=-(w / 2)\left(x L-x^{2}\right)
$$

In this case, a uniform distributed load w loads the upper surface of a beam with elastic modulus $\mathrm{E}$ and moment of inertia I, from length $\mathrm{x}=0$ to $\mathrm{x}=\mathrm{L}$, the beam length. Deflection is measured in the direction $\mathrm{y}$ at the free end of the beam, $\mathrm{x}=\mathrm{L}$.

We use a series of three different finite element codes, for a total of $\mathrm{N}_{\mathrm{s}}=6$ sets of results. The third series is also taken from the $2^{\text {nd }}$ Verification Suite, and is a comparison on $\mathrm{N}_{\mathrm{g}}=4$ grids (the minimum number of grids for Methods \#5 to \#10). The problem is beam bending, with an exact solution once again known. This time the same three finite element codes are compared, on stress and deflection (giving an additional $\mathrm{N}_{\mathrm{s}}=6$ sets of grids), but with a brick element mesh. Altogether, we have $\mathrm{N}_{\mathrm{s}}=18$ sets of grid convergence studies, with $\mathrm{N}_{\mathrm{g}}=7$ or $\mathrm{N}_{\mathrm{g}}=4$ grids for each.

How will we evaluate the usefulness of each of our 10 methods for estimating $U_{\text {sver }}=U$, our solution verification contribution to uncertainty at (1-sigma in this case) confidence? For each of our $\mathrm{N}_{\mathrm{s}}=18$ sets of grid convergence studies, we will, in the end, generate an estimate (Eqn. [4]) of $\mathrm{U}_{\text {sver }}=\mathrm{U}$; our estimate of the uncertainty at 1-sigma of our fine-grid solution $\mathrm{F}_{\mathrm{fg}}{ }^{*}$, compared to our estimate $\mathrm{F}_{\mathrm{o}}{ }^{*}$ of the exact solution. Since all of the $\mathrm{N}_{\mathrm{s}}=18$ sets have known analytical solutions, we can compare this $\mathrm{U}_{\text {sver }}$ to the actual difference $\mathrm{E}_{\text {actual }}$ between the code-produced fine grid solution $\mathrm{F}_{\mathrm{fg}}$ and the known solution $\mathrm{F}_{\mathrm{o}}$. If we claim 1-sigma confidence assuming normal distributions, and we are neither too conservative nor too optimistic, we should find that about $68 \%$ of the time $(12 / 18)$ the $U_{\text {sver }}$ estimate should enclose $E_{\text {actual }}$. In about 6 of 18 cases, we should find that $U_{\text {sver }}$ does not enclose $E_{\text {actual. }}$. Similarly, if we use the 2-sigma estimate of $U_{\text {sver }} 2 \sigma$, we should find that in $95 \%$ of cases (about $17 / 18$ ) $U_{\text {sver }}$ should enclose $E_{\text {actual }}$. In about 1 of 18 cases, $E_{\text {actual }}$ should be larger than $U_{\text {sver }} \mid 2 \sigma$. We define our Solution Verification Method Reliability $\mathrm{R}_{\mathrm{sm}}$ as

$\mathrm{R}_{\mathrm{sm}}=1 .-$ | Fraction inside, Method Estimate - Fraction inside, expected $\mid$

That is, at 1-sigma for $\mathrm{N}_{\mathrm{s}}=18$, we expect 12 of the series to have $\mathrm{U}_{\text {sver }}>\mathrm{E}_{\text {actual, }}$ or Fraction Inside, Expected=12/18=.667. If our Method (\#1 thru \#10) assesses all $18 \mathrm{U}_{\text {sver }}>\mathrm{E}_{\text {actual, }}$, then Fraction Inside, Method = 1.000 and $\mathrm{R}_{\mathrm{sm}}=1 .-|1.000-0.667|=67 \%$. If our Method assesses $12 \mathrm{U}_{\mathrm{sver}}>\mathrm{E}_{\text {actual }}$, then $\mathrm{R}_{\mathrm{sm}}=1 .-|.667-.667|=100 \%$.

If our method assesses only $9 \mathrm{U}_{\mathrm{sver}}>\mathrm{E}_{\text {actual, }}$, then $\mathrm{R}_{\mathrm{sm}}=1 .-|.500-.667|=83.3 \%$. For a method that is neither too optimistic (risk induced due to non-conservatism) nor too pessimistic (too risk-averse) we want $R_{\text {sm }}$ to be as close as possible to $100 \%$. As just stated, we would expect at $2 \sigma$ (95\% confidence) that about $5 \%$ of the time, or in about $1 / 18$ cases, $E_{\text {actual }}$ would be larger than $\mathrm{U}_{\text {sver }} \mid 2 \sigma$. It is worth noting that our definition of Method Reliability means that even if we find, at $2 \sigma$, that $E_{\text {actual }}$ is larger than $U_{\text {sver }} \mid 2 \sigma 0 / 18$ or $2 / 18$ times instead of our expected $1 / 18$ time, the Method Reliability will only be lowered by $6 \%$ (one in 18). Naturally, a sample size of $\mathrm{N}_{\mathrm{s}}>>18$ would be better, but our sample size of $\mathrm{N}_{\mathrm{s}}=18$ will make our points without excessive truncation artifacts due to small integer sample size. This definition of "Method Reliability" is similar, though not identical, to the development and usage given by Urbina, Paez, et $\mathrm{al}^{16}$. Figure 4 shows the results on our $\mathrm{N}_{\mathrm{s}}=18$ grid sets, for the 10 methods $\# 1$ through \#10. All 10 
of the Methods show fairly high Method Reliability $\mathrm{R}_{\text {sm }}$, except for the use of Method \#2, with GCI=1.25 as a "95\% confidence" estimate. We realize that there is a large database of CFD solutions (perhaps mostly smooth and monotonic) that supports the use of Method \#2. However, for our contrived and mechanics example $\mathrm{N}_{\mathrm{s}}=18$ sets (most of which were non-smooth), the use of GCI $=1.25$ is much closer to a $68 \%$ confidence estimate than $95 \%$. A larger sample set is needed to draw any general conclusions in this regard, but we suspect that this observation is due perhaps to the use of solid mechanics examples, but due most likely toward our intentional selection of nonsmooth grid convergence results. It is important to note that we did not allow values of exponent $\mathrm{p}>2$ in our Method \#1-\#10 fitting procedures, since none of our examples had formulations that would enable convergence or order $\mathrm{p}>2$. Sometimes fitting methods in solution verification for non-smooth grid convergence results can give spurious values ${ }^{9}$ of $p>>2$, or $p$ larger than the theoretical value of the formulation. We attempted to avoid this situation by not allowing any fits with $p>2$. Roache has suggested ${ }^{17}$ that in the GCI procedures, it is best to use the lesser of the observed $\mathrm{p}$ and the theoretical $\mathrm{p}$ of the numerical algorithm being used. We agree and would extend the suggestion to the entirety of Method \#1 to Method \#10.

The "Method Reliability" shown in Fig. 4, although encouraging, cannot be used to select or even suggest the use of some of Method \#1-\#10 versus the others. Perhaps an extension of our data set beyond $\mathrm{N}_{\mathrm{s}}=18$ will reveal more about the method reliabilities.

A measure of robustness of each of the Methods \#1 to \#10 can be given by generating a variant of modification of the reduced Chi-Square $\left(\mathrm{X}_{\mathrm{v}}^{2}\right)$ style statistic ${ }^{18}$. We use a modified form of $\mathrm{X}^{2}{ }_{\mathrm{v}}{ }^{*}$ as:

$\mathrm{X}_{\mathbf{v}^{2}}{ }^{*}=\quad \begin{array}{ll}\mathrm{Ns}=18 \\ \sum_{\mathrm{I}=1}\left[\left(\mathrm{U}_{\mathrm{sver}}\right) /\left(\mathrm{F}_{\mathrm{fg}}-\mathrm{F}_{\mathbf{o}}\right)\right]^{2} /\left(\mathrm{N}_{\mathbf{s}}=18\right)\end{array}$

We stress that our $\mathrm{X}^{2}{ }_{\mathrm{v}}{ }^{*}$ is a modified form of $\mathrm{X}^{2}$, in that we are not attempting to use model free parameters to remove bias as is commonly done leading to terms such as (N-1) in the denominator of standard deviation. A high value for this $\mathrm{X}_{\mathrm{v}}^{2}{ }^{*}$ indicates a solution verification method that is too conservative; $\mathrm{U}_{\text {sver }}$ is in general much wider than Eactual. We took this value of $\mathrm{X}^{2} \mathrm{v}^{*}$ for each method, but also added the standard deviation of the $\mathrm{X}^{2}{ }_{\mathrm{v}}{ }^{*}$ ratio for each of the $\mathrm{Ns}=18$ sets. Ideally, if $\mathrm{U}_{\text {sver }}=\mathrm{E}_{\text {actual }}$ in each of the $\mathrm{N}_{\mathrm{s}}=18$ series, we would have $\mathrm{X}^{2}{ }_{\mathrm{v}}{ }^{*}=1.00$ and the standard deviation $\mathrm{S}\left(\mathrm{X}_{\mathrm{v}}{ }_{\mathrm{v}}{ }^{*}\right)=0.00$ so $\mathrm{X}_{\mathrm{v}}{ }^{*}+\mathrm{S}\left(\mathrm{X}_{{ }_{\mathrm{v}}}{ }^{*}\right)=1.0+0.0=1.0$. The actual values are shown in Figure 5. This figure is a measure of the combination of over-conservatism and ambiguity (standard deviation) of the individual and composite $\mathrm{X}^{2}{ }_{\mathrm{v}}{ }^{*}$ assessment of each method. Considering that the Method Reliability $\mathrm{R}_{\mathrm{sm}}$ numbers were quite good, we might expect these $X^{2}{ }^{*}{ }^{*}$ values to be lower than they are. We intentionally picked grid studies with oscillations in both exponent $p$ and even output quantity $F_{i}$, which may explain why we have good $R_{s m}$ but $X^{2}{ }_{v}{ }^{*}>>$ 1 in general, and why there is no apparent correlation between $R_{s m}$ and $X^{2} v^{*}$.. We intend to add more data sets to our study and compare smooth convergence studies with oscillatory ones. In any case, lower $\mathrm{X}^{2}{ }_{\mathrm{v}}{ }^{*}$ numbers are better, so we can see that while most of the Methods \#1 to \#10 gave high Method Reliability $\mathrm{R}_{\mathrm{sm}}$, Method \#1 to \#5 give unpredictable results for any given individual series. Hence we highly recommend the use of one of more of Method \#6 to Method \#10 for the most reliable and robust estimates of $\mathrm{U}_{\text {sver. }}$.

Mostly, we stress that the majority of Method \#1 to \#10 for a solution verification uncertainty at confidence estimate show high method reliability $\mathrm{R}_{\mathrm{sm}}$, and therefore we urge the use of at least a few of these methods as opposed to no estimate at all. 


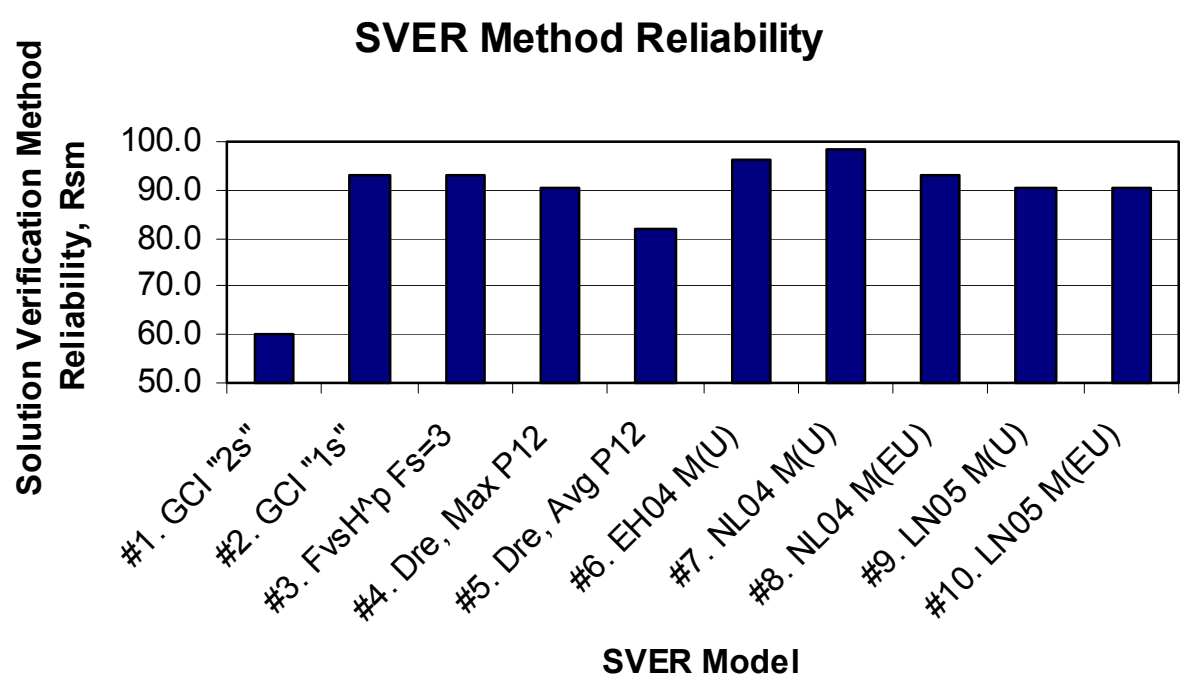

Figure 4. Solution Verification Method Reliability Rsm, for the 10 Methods \#1 to \#10 on the 18 grid set series discussed.

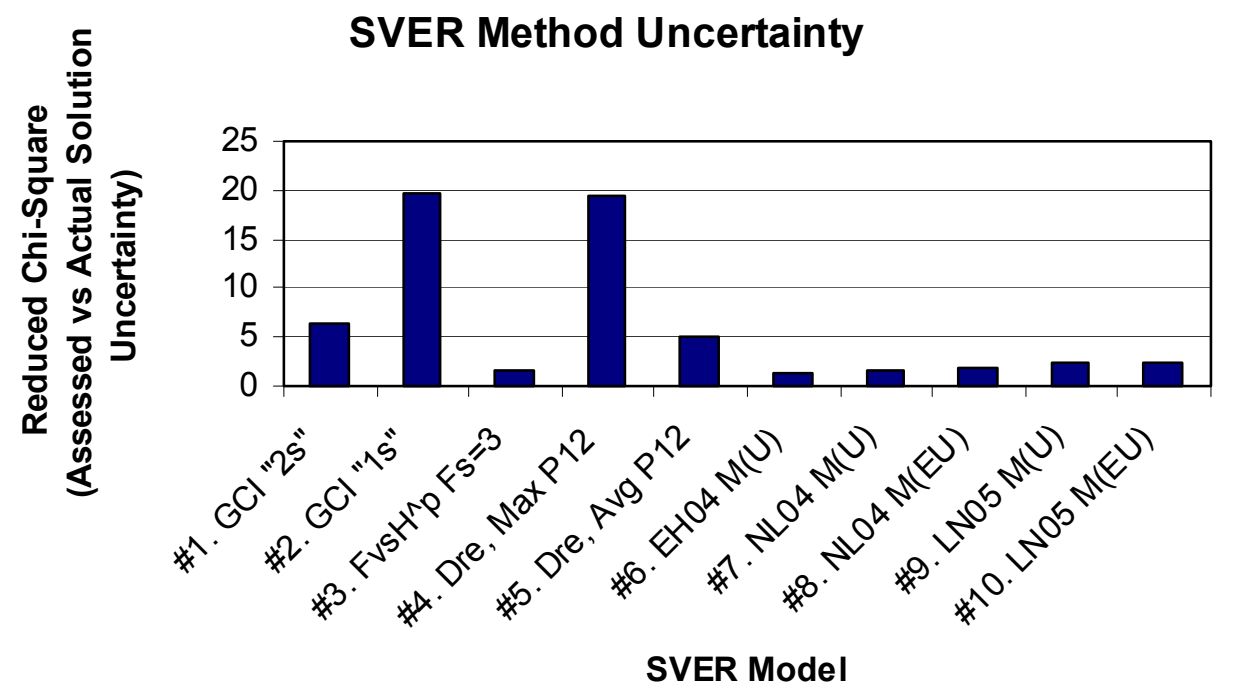

Figure 5. Reduced Chi-Square measure of Solution Verification Method overconservatism added to scatter in conservatism. Values closer to one are best; high values are either systematically or erratically overconservative. 


\section{Linking Solution Verification to Model Validation}

We used Method \#1 to Method \#10 on the grid convergence study data of Nitta and Logan ${ }^{9}$, and on a few of the examples from Eça and Hoekstra ${ }^{8}$. These grid convergence studies gave either non-smooth or even non-monotonic results, making them ideal for a test of the different methods. We confirmed that in general, it is best to use several of the Methods as part of a grid convergence study, and in particular we recommend running several variants of Method \#1 to Method \#10. We observed several cases where the inclusion of the prediction extrapolation parabola (Method \#9 and \#10) gave added stability in avoiding a spurious fit. We also observed instances where minimizing $\left(\mathrm{E}+\mathrm{U}_{\mathrm{s}}\right)$ as in Method $\# 8$ and $\# 10$, avoided optimizing to a spurious solution ${ }^{9}$ with apparent super-convergence. That is, we have seen instances where Method \#7 or Method \#9 would indicate a spurious best-fit $\mathrm{p}=3$, whereas Method $\# 8$ or Method \#10 would show a best-fit with $\mathrm{p}<<2$. However, none of the methods so far are foolproof, so we recommend several different combinations. Eça and Hoekstra ${ }^{8}$ offered one additional simple, and perhaps close to bounding, estimate of $\mathrm{U}_{\text {sver }}$ for extremely difficult non-monotonic grid sets, and we give an example here to illustrate:

Consider the grid convergence results shown in Figure 6. These are contrived numbers, but very close to situations we have seen in nonlinear large deformation mechanics problems. Of note is that only $\mathrm{N}_{\mathrm{g}}=3$ grids are available. However, in this case, we make the reluctant assumption that for similar codes, physics, algorithms, and problem application, we might assume the same convergence order " $p$ " for all four grid series. We now have only $\mathrm{K}=2$ free parameters $\left(\mathrm{F}_{\mathrm{o}}{ }^{*}\right.$ and $\left.\alpha\right)$, so we need a minimum of $\mathrm{N}_{\mathrm{g}}=3$ grids. With Method $\# 10$, the LN05eu method, we are able to generate solution verification uncertainty at confidence estimates that are stable and compare well with an "Emergency Method \#11" as proposed by Eça and Hoekstra ${ }^{8}$. For what we call Method \#11, Eça and Hoekstra propose to take the largest magnitude of observed difference in two adjacent grids, and triple that value to obtain an estimate of the $\mathrm{U}_{\text {sver }}$ uncertainty. We find that our Method \#10 bounds their number with 1.00 sigma and 1.65 sigma analyses. All the methods tell us one very clear thing about this analysis: Three grids $\mathrm{N}_{\mathrm{g}}=3$ is rarely enough for a credible assessment of $U_{\text {sver }}$, even if we assume the same p-value to make the analysis even possible. Furthermore, with grid results as shown here, the message is simply that more work is needed. If a number must be had with only the work shown in Fig. 6-7, the value of $U_{\text {sver }}$ shown should be sufficiently large to avoid any misinterpretation. In this case, an assessment that the solution verification uncertainty is $50 \%$ to $100 \%$ of the quantity of interest would convey the proper message; that this model would only give us a converged answer "within a factor of two or so".

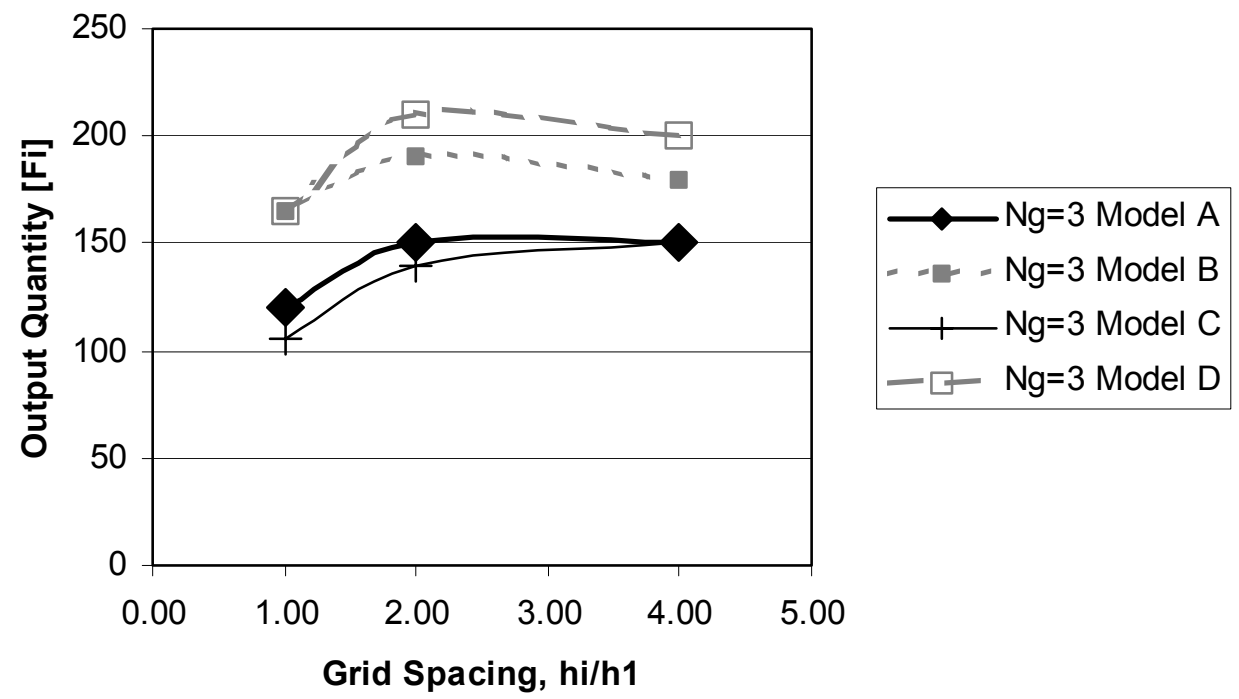

Figure 6. Special case example for $\mathrm{N}_{\mathrm{g}}=3$; assume same $\mathbf{p}$ for all four grid series leaving $\mathrm{K}=2$. Convergence for the sparse $\mathbf{N}_{\mathrm{g}}=3$ ranges from oscillatory to ambiguous. 


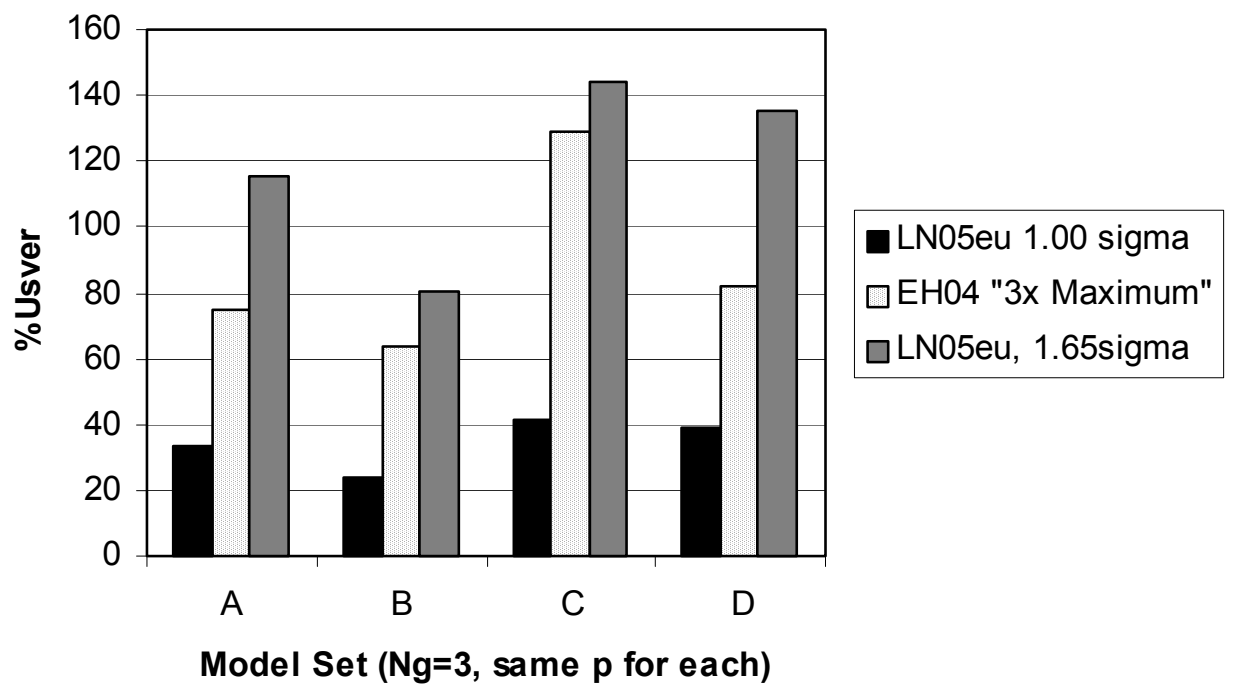

Figure 7. Special case example for $\mathbf{N}_{\mathrm{g}}=3$; assume same $\mathbf{p}$ for all four grid series leaving $\mathrm{K}=2$. Uncertainty estimates at 1-sigma are $24 \%$ to $40 \%$ using Method \#10 at 1-sigma. Eça and Hoekstra " $3 x$ max difference" gives uncertainties of $60 \%$ to $130 \%$, about equal to Method \#10 at 1.65 sigma. Either way, the message has been sent; this situation heeds help.

Having described 10 methods for quantification of a term $\mathrm{U}_{\text {sver }}$ as $\mathrm{U} \mid \mathrm{C}$, an uncertainty at an assessed statistical confidence e.g. $\mathrm{C}=95 \%$, we now proceed to link this result into a quantitative validation statement that results from $[C]$ of our "ABCD" $V \& V$ process.

First of all, we offer a suggestion on how to proceed if our suggestion of trying more than one of Method \#1 to \#10 is used. If you are ambitious enough to try more than one of Method \#1 to Method \#5, and you see large differences between the $\mathrm{U}_{\text {sver }}$ estimates, we suggest using one or more of Method \#6 to Method \#10. Methods \#6-\#10 should give very similar estimates of $U_{\text {sver }}$. If they do not, we suggest using the largest of the $U_{\text {sver }}$ from Method \#6-\#10, while investigating the causes for the large discrepancies between the methods. If all else fails and you run out of time, use the "Emergency Method \#11" discussed and illustrated in Figs. 6-7.

We have now assessed an uncertainty at confidence $\left(\mathrm{U}_{\text {sver }} \mid \mathrm{C}\right)$ for the grid convergence portion of solution verification. We have previously provided examples of how this $\left(\mathrm{U}_{\mathrm{sver}} \mid \mathrm{C}\right)$ term is used in proceeding from solution verification to validation, reliability, risk, and finally the generation of Benefit / Cost Ratios (BCRs) for future actions ${ }^{9}$. The essential steps linking these processes are:

- Plot the quantity of interest versus grid spacing

- Use a mixture of Method \#1 to Method \#11 to generate the solution verification uncertainty at confidence, $\mathrm{U}_{\text {sver }} \mid \mathrm{C}$. This is "Step B" of the "ABCD" V\&V process we outlined to begin this work.

- If your analysis is assessed to be low risk, or if you are confident that the conditions for the use of the GCI are valid for your analysis, then the GCI (Method \#1) with $\mathrm{N}_{\mathrm{g}}=3$ or even $\mathrm{N}_{\mathrm{g}}=2$ offers a fast assessment of $\mathrm{U}_{\text {sver }} \mid \mathrm{C}$ that may indicate whether more advanced methods (Method \#6-\#10) and more grids $\mathrm{Ng} \geq 4$ are needed.

- In general however, as illustrated in our last example, three grids $\mathrm{N}_{\mathrm{g}}=3$ is rarely enough for a credible assessment of $\mathrm{U}_{\text {sver, }}$, even if we assume the same $\mathrm{p}$-value to make the analysis even possible.

- Use this $\mathrm{U}_{\text {sver }} \mid \mathrm{C}$ value as a mean value (MV) contribution to continue to Model Validation Process "Step C and $\mathrm{D}$ " of "ABCD" $\mathrm{V} \& \mathrm{~V}$. (There are methods more elaborate than including $\mathrm{U}_{\text {sver }} \mid \mathrm{C}$ as a mean value contribution, and if $\mathrm{U}_{\mathrm{sver}} \mid \mathrm{C}$ is large enough to have an impact on your validation statement about the model, the inclusion of $\mathrm{U}_{\mathrm{sver}} \mid \mathrm{C}$ as a mean value (MV) term may not be adequate. Methods more advanced than MV 
are sometimes considered as integral with the type of reliability analysis ${ }^{3,19}$ to follow).

- Combine the total and components of uncertainty at confidence from validation with system requirements, to generate a reliability $\% \mathrm{R},(0 \%<\% \mathrm{R}<100 \%)$ for the model of the system (at a given assessed percent confidence level, $0 \%<\% \mathrm{C}<100 \%$ ).

- Combine these measures of model+system reliability at confidence with a consequence assessment of success and failure of the system, leading to quantitative risk and potential for risk mitigation or reduction.

- Assess the Benefit/Cost Ratio BCR of the opportunities for risk mitigation or reduction.

The measures for risk mitigation or reduction might include taking more experimental data or improving model physics $^{5}$, or perhaps buying more compute capability ${ }^{9}$ to allow the chance to lower solution verification uncertainty.

\section{Conclusions: Linking Solution Verification to Validation, Risk, Reliability, and Benefit/Cost Ratio}

We have taken our discussion from the first step in a grid convergence study (plotting the quantity of interest versus grid spacing) to the point where an input term for validation is generated. This term is an uncertainty at confidence, $\mathrm{U}_{\text {sver }} \mid \mathrm{C}$. We described 5 relatively simple methods to assess $U$, and 5 more complex methods, with 1 emergency method as a backup. Of these 10 (plus 1 for emergencies) methods for assessing the uncertainty term $\mathrm{U}_{\text {sver }}$ for solution verification, we suggest that more than one be used. The choice, and subsequent action, may depend on the discrepancy between the value of $U_{\text {sver }}$ obtained from each method, as well as the risk involved in using the simulation being considered. The values of $U_{\text {sver }}$ we have obtained range, at 1-sigma confidence, anywhere from less than $5 \%$ of the output quantity to as much as $100 \%$ of the output quantity. It is not unusual to see values of $U_{\text {sver }}$ at 1-sigma of $30 \%$ of the output quantity when grid convergence studies are non-monotonic in the output quantity. We do not know of any method to assess grid convergence uncertainty that will circumvent the reality that nonsmooth grid convergence results are going to lead to large values for the solution verification uncertainty term.

We suggest that there is no unique method to assess solution verification uncertainty, especially for non-smooth grid convergence results. We have presented 10 such methods, and suggest using the largest value of Usver so obtained while further investigation is pursued if warranted. We have structured each method with the goal of a $U_{\text {sver }}$ term that links directly into a quantitative validation process, enabling quantification of assessed risk and the potential for reduction in assessed risk with more work in solution verification. This linkage is important, because if we can show the value (in terms of risk and benefit / cost ratio) of solution verification, perhaps it will be easier to justify the effort spent on the process. Since there is a balance between the amount of effort (cost) spent on V\&V of a model, and the amount of value (reduced assessed risk) that can be gained, we have presented a method to quantify the contribution of solution verification to this balance.

Developing our computational models in a systems engineering context will enable us to balance both of these ends of the scale, and defend our determination of this balance. However, as we show in the analysis, the balancing point depends on the details and fidelity of the quality of the codes, solution verification issues, referent data and model validation status, consequences leading to risk assessments, and the all important balance of the BCR in determining when "better has become the enemy of good enough".

\section{Acknowledgements}

This work was performed under the auspices of the U.S. Dept of Energy by Lawrence Livermore National Laboratory under contract W-7405-Eng-48. The authors are grateful for their interaction with a large community in these fields. In particular, we wish especially to thank Luis Eça, Patrick Roache, Tim Trucano, Henry Hsieh, Ben Thacker, David Sam, Tom Paez, for the discussions that motivated this work on solution verification and its ultimate linkage to the decision process. 


\section{References}

${ }^{1}$ Logan, R.W. and Nitta, C.K., "Verification \& Validation: Process and Levels Leading to Qualitative or Quantitative Validation Statements", LLNL UCRL-TR-200131 Oct. 2003.

${ }^{2}$ Easterling, R.C., "Statistical Foundations for Model Validation: Two Papers", SAND2003-0287, Sandia National Laboratories, Feb 2003.

${ }^{3}$ Nitta, C.K. and Logan, R.W., "Qualitative and Quantitative Linkages from V\&V to Adequacy, Certification, Risk, and Benefit / Cost Ratio", UCRL-TR-205809, Oct 2004.

${ }^{4}$ Oberkampf, W.L., Trucano, T.G., and Hirsch, C., "Verification, Validation, and Predictive Capability in Computational Engineering and Physics", Proc. Foundations 02, Jo.hns Hopkins Univ., Laurel, MD, Oct 2002..

${ }^{5}$ Logan, R.W., Nitta, C.K., and Chidester, S.K., "Risk Reduction as the Product of Model Assessed Reliability, Confidence, and Consequence", LLNL UCRL-AR-200703, Nov. 2003.

${ }^{6}$ Roache, P.J., Verification and Validation in Computational Science and Engineering, Hermosa Publishers, Albuquerque, NM, 1998.

${ }^{7}$ Richardson, L.F., "The Deferred Approach to the Limit", Trans. Royal Society of London Series A, Vol. 226, p. 299.

${ }^{8}$ Eça, L. and Hoekstra, M., "An Uncertainty Estimation Exercise with the Finite-Difference and Finite-Volume Versions of PARNASSOS", Proc. Workshop on CFD Uncertainty Analysis, 21-22 Oct 2004, Instituto Superior Technico, Lisbon, Portugal.

${ }^{9}$ Nitta, C.K. and Logan, R.W., "Solution Verification Linked to Model Validation, Reliability, and Confidence", UCRL-CONF-206544, Sep 2004.

${ }^{10}$ ASME, “Test Uncertainty”, Performance Test Codes PTC 19.1, an American National Standard, ASME, New York, NY, Dec 1998.

${ }^{11}$ Roache, P.J., "'"Conservatism of the GCI in Finite Volume Computations on Steady State Flow and Heat Transfer", ASME Journal of Fluids Engineering, Vol. 125, No. 4, July 2003, pp. 731-732.

${ }^{12}$ Moore, D.S. and G.P. McCabe, Introduction to the Practice of Statistics, W.H. Freeman \& Co., 1989.

${ }^{13}$ Harrison, J.L. and Conway, R., "Second Verification Test Suite for ASCI Codes for the Complex", LLNL report CODTU-2003-1225, Rev. 1, Oct 2003.

${ }^{14}$ Logan, R.W. and Nitta, C.K., "Verification \& Validation (V\&V) Methodology and Quantitative Reliability at Confidence (QRC): Basis for an Investment Strategy", UCRL-ID-150874, Nov 2002.

${ }^{15}$ Sam, D.D., Murty, S.S., Perfect, S.A., Castano, D., and Pott, J., "First Verification Test Suite for ASCI Codes for the Complex", LLNL report CODTU-2002-0113, Jan 2002.

${ }^{16}$ Urbina, A., Paez, T.L., Gregory, D.L., and Resor, B.R., "Response Comparisons for Mechanical Joint Model Validation", Proc. $9^{\text {th }}$ ITEA Modeling \& Simulation Conference, Las Cruces, NM, Dec 2003.

${ }^{17}$ Roache, P.J., private communication, March 2005.

${ }^{18}$ Bevington, P.R. and Robinson, D.K., "Data Reduction and Error Analysis for the Physical Sciences", McGrawHill, 1969.

${ }^{19}$ Hsieh, S.R and Sam, D.D., "Virtual Proving Ground for Assessing Reliability and Uncertainty”, UCRL-CONF-205448, July, 2004. 